\title{
Pubertal Growth in Chronic Renal Failure
}

\author{
FRANZ SCHAEFER, CHRISTOFFER SEIDEL, ANGELIKA BINDING, THEO GASSER, \\ REMO H. LARGO, ANDREA PRADER, AND KARL SCHÄRER
}

\begin{abstract}
Division of Paediatric Nephrology, University Children's Hospital, Heidelberg (F.S., C.S. K. S.]; Institute for Statistics and Applied Mathematics, University Heidelberg [A.B.J: and Institute for Mental Health, Mannheim. Federal Republic of Germany [T.G.], and University or Children's Hospital, Ziorich, Switzerland [R.H.L., A.P.]
\end{abstract}

\begin{abstract}
We evaluated the growth records of 15 boys and 14 girls who developed end-stage renal failure before or during puberty and who were regularly followed from the onset to the end of their pubertal growth spurt. Height data were smoothed by using the kernel estimation method. Mean values for age, height, and height velocity at defined points of the pubertal growth period were compared with those of normal children entering puberty both at an ayerage and late age. The start of the pubertal growth spurt was delayed by $2.5 \mathrm{y}$ in both sexes. Its duration and intensity were significantly reduced. Mean pubertal height gain was $17.3 \mathrm{~cm}$ in boys and $13.9 \mathrm{~cm}$ in girls, i.e. 58 and $48 \%$ of that observed in the late maturing control group. Mean height at the onset of the pubertal spurt in the patients was the same as that in the late maturing healthy girls and 1.0 SD below that of corresponding boys. During the pubertal growth spurt, mean height declined to -2.9 $\mathrm{SD}$ in boys and $-2.3 \mathrm{SD}$ in girls. Although skeletal maturation was increasingly retarded, we did not observe accelerated growth velocity during late puberty. Our data indicate that most patients reaching end-stage renal failure before or during puberty irreversibly lose growth potential during this period. Renal transplantation did not consistently improve pubertal growth. (Pediatr Res 28: 5-10, 1990)
\end{abstract}

\section{Abbreviations}

CRF, chronic renal failure

HD, regular hemodialysis

TP, renal transplantation

Scr, serum creatinine

MHV, minimal prespurt height velocity

PHV, peak height velocity

EHV, end-point height velocity

SDS, standard deviation score

CRF is frequently associated with growth retardation, often leading to permanent stunting despite optimal therapy including successful TP $(1,2)$. The degree of growth retardation is influenced by the primary kidney disease, the clinical course, malnutrition, abnormalities of acid-base and electrolyte status, and endocrine growth factors. It has been emphasized that, during the critical period of infancy, there is a rapid loss of growth potential that is rarely regained in later childhood (3). Our report presents data suggesting that the pubertal period is similarly

Received July 5, 1989; accepted February 6, 1990

Correspondence and reprint requests: Dr. F. Schaefer, Division of Pediatric Nephrology, University Children's Hospital, Im Neuentheimer Feld 150, D-6900 Heidelberg, FRG

Supported by Deutsche Forschungsgemeinschaft Grant No. Scha 169/5-1. sensitive to the growth retarding effects of uremia and its treatment.

Previous investigations on pubertal growth under pathologic conditions have generally been hampered by methodologic problems (4). In our report, we introduce the kernel estimation technique as a new method that allows objective definition of characteristic points of the pubertal growth spurt, thus facilitating analysis and comparison with control data $(5,6)$.

\section{MATERIALS AND METHODS}

Study population. Data were reviewed from 15 boys and 14 girls with CRF who had been regularly observed at 3- to 6-month intervals at the Heidelberg University Children's Hospital between 1968 and 1988. The patients had the following primary renal disorders: focal-segmental glomerulosclerosis $(n=6)$, obstructive uropathy $(n=6)$, renal hypoplasia $(n=5)$, nephronopthisis $(n=4)$, Schönlein-Henoch nephritis $(n=2)$, rapidly progressive glomerulonephritis $(n=2)$, others $(n=4)$.

The patients were followed from 0.5 to 11.8 (median 3.3 ) y before the onset of the pubertal growth spurt, as defined by the MHV (see below), until pubertal growth had ceased. Median ages (range) at initial and at final observation were $9.3(0.5-11.8)$ and $20.2(16.3-25.2)$ y, respectively. At initial observation, 24 patients did not manifest any clinical signs of pubertal development, and two boys and three girls had reached Tanner pubic hair (PH) and/or genital (G) or breast stage 2. At final observation, all patients had attained puberty stage 4 or 5 except one boy who was stage $\mathrm{PH} 3 / \mathrm{G} 3$. Hand epiphyses were closed in all patients except for four boys with bone ages of 16.3-17.5 y and two girls with bone ages of 14.6 and $15.6 \mathrm{y}$, respectively.

The onset of CRF was defined by the first record of an increased Scr level $\geq 1.3 \mathrm{mg} / \mathrm{dL}$. At first observation, 27 patients had Scr levels ranging from 0.4 to $9.2 \mathrm{mg} / \mathrm{dL}$ (median 2.2) and two were undergoing HD. The treatment modalities at defined points of the growth spurt are shown in Figure 1. At last observation, 21 patients had a functioning TP (median Scr $1.9 \mathrm{mg}$ / $\mathrm{dL}$, range $0.9-6.0$ ), seven were on $\mathrm{HD}$ after TP failure, and one had never received a TP.

After TP, patients received immunosuppression with azathioprine and (usually daily) methyl-prednisolone or prednisone. The mean methyl-prednisolone equivalent dosage given was 6.1 $\pm 0.7 \mathrm{mg} / \mathrm{m}^{2} / \mathrm{d}$. Cyclosporin A was given to only one patient.

Analysis of growth. Height was measured with standard methods (7) by trained personnel using a Holtain stadiometer (Holtain Ltd., Crymych, Dyfed, UK). Height was usually recorded at 3mo intervals; thus, a total of 959 data points were available for analysis. Pubertal status was evaluated according to Tanner (8) by the examining physician. Hand $x$-rays were obtained at approximately $6-\mathrm{mo}$ intervals. Bone age was determined $(n=180)$ according to the TW2 method (9) by a trained observer with an intraobserver variation of \pm 0.5 y $(95 \%$ confidence limit) (7).

To minimize the influence of measurement errors, height data were smoothed by the kernel estimation method $(5,6)$. This is a 
mathematical procedure applying a moving weighted average with raw data. It estimates a function at time $t$ by

$$
\hat{\mathrm{r}}(\mathrm{t} ; \mathrm{b})=\Sigma \mathrm{g}_{i}(\mathrm{t}) \cdot \mathrm{y}_{\mathrm{i}}
$$

where $y_{i}=$ measurement at time $t_{i}, g_{i}(t)=1 / b \cdot \int_{s_{j-1}}^{s_{i}} w\left(\left(t_{i}-u\right) /\right.$ b)du, $\mathrm{s}_{\mathrm{i}}=\left(\mathrm{t}_{\mathrm{i}+1}-\mathrm{t}_{\mathrm{i}}\right) / 2$, w $=$ kernel (or wt) function, $\mathrm{b}=$ bandwidth or smoothing parameter.

In contrast to parametric regression methods (10), kernel estimation makes no assumption on the shape of the true curve. This principle is essential in the evaluation of pathologic growth curves as in CRF, where abrupt changes due to clinical events may be expected. Moreover, choice of appropriate weighting functions allows direct estimation of derivatives of the height curve, i.e. of the corresponding height velocity and acceleration curve. The degree of smoothing was chosen data-adaptively by minimizing the mean square error (Gasser T, Köhler W, Kneip A, unpublished manuscript).

The point at which the derived smooth height velocity curve reached a maximum during puberty was defined as the $\mathrm{PHV}$. It represents the growth increment during a time period spanning six mo to either side of this time point. The preceding minimum of the height velocity curve was defined as MHV and used as the starting point of the pubertal growth spurt (6). The end of the pubertal growth spurt was defined as the age at which the late ht velocity curve permanently declined below $1 \mathrm{~cm} / \mathrm{y}(\mathrm{EHV})$. The pubertal growth period was defined by the time interval between MHV and EHV.

The individual growth curves obtained by the kernel estimation procedure were centered on MHV, PHV, and EHV. For this purpose, we used a synchronization program that transforms the time scale of each individual curve monotonously to align the characteristic points with the respective means (Kneip A, Gasser T, unpublished manuscript).

Control data were from the First Zurich Longitudinal Growth Study, comprising 232 healthy children ( 120 boys, 112 girls) who were followed longitudinally throughout the growth period (control group A) (13). Because the growth spurt characteristics depend on the "tempo" of the maturing process and the patients under study represent a late maturing population, we analyzed separately those 70 children ( 35 boys, 35 girls) in the Zurich Longitudinal Growth Study who had the latest pubertal growth spurt (control group L).

Mean values for MHV, PHV, and EHV and for the ages at which these parameters were reached were calculated for the CRF patients and both control groups. Data are given as mean $\pm \mathrm{SD}$. Because the distributions of height and ages in the control groups were approximately gaussian, the degrees of deviation from the normal means were expressed as SDS. The definitions of MHV, PHV, and EHV allowed calculation of conditional SDS related to the respective points of the pubertal growth spurt rather than to chronologic age or bone age. Differences between

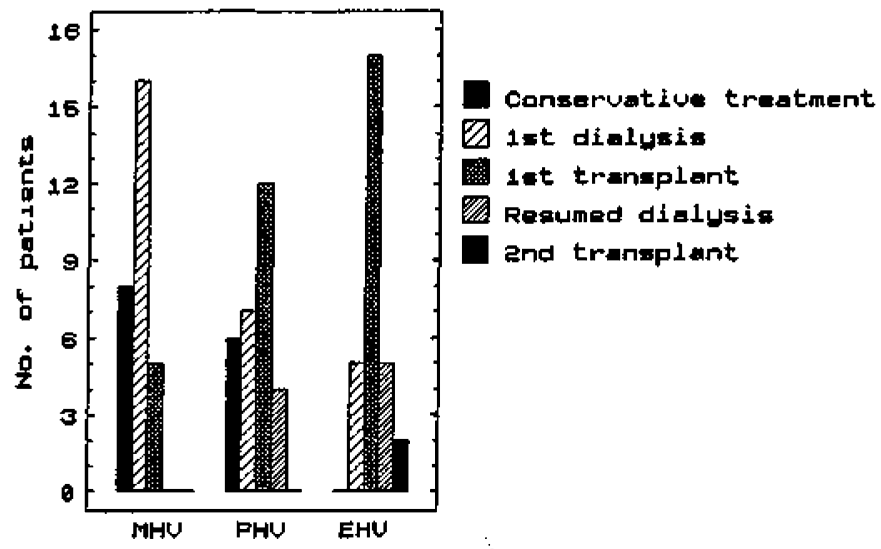

Fig. 1. Treatment of 29 children with CRF at defined points of pubertal growth. the patients and the control group data were checked with paired $t$ test for statistical significance.

\section{RESULTS}

All CRF patients except one girl exhibited a distinct pubertal growth spurt demonstrated by an upward turn of the height velocity curve. The spurt amplitude was less than $1 \mathrm{~cm}$ in three boys and two girls. The synchronized mean height velocity curves of boys and girls are shown in Figures 2 and 3, respectively.

Mean MHV occurred at $13.4 \mathrm{y}$ in boys and $12.1 \mathrm{y}$ in girls, which corresponds to a delay in the CRF patients by about 2.5 SD compared with control group A patients (Table 1). Age at PHV was slightly less delayed than MHV in both sexes, and the mean delay of EHV was even less than that of PHV. The delay of MHV, PHV, and EHV $(p<0.001)$ was significant when compared with control group A. This delay did not reach significance when compared with control group L, except for MHV. The mean duration of the pubertal growth period was $5.5 \pm 1.6$ $y$ in boys and $4.4 \pm 1.7 \mathrm{y}$ in girls, which was shorter than in children of control groups A (both sexes, $p<0.001$ ) and L (boys, $p<0.02$; girls, $p<0.01$ ).

The mean height and height velocities attained by the CRF patients at the defined points of the pubertal spurt compared with the two control populations are given in Table 2. Although age-related height SDS at first observation was grossly reduced in the CRF patients to -2.2 and $-1.8 \mathrm{SDS}$ in boys and girls, respectively, the mean heights attained at MHV were only 1.0 SD below the mean of control group $L$ boys and at the mean of late maturing girls. At the later points of the pubertal growth

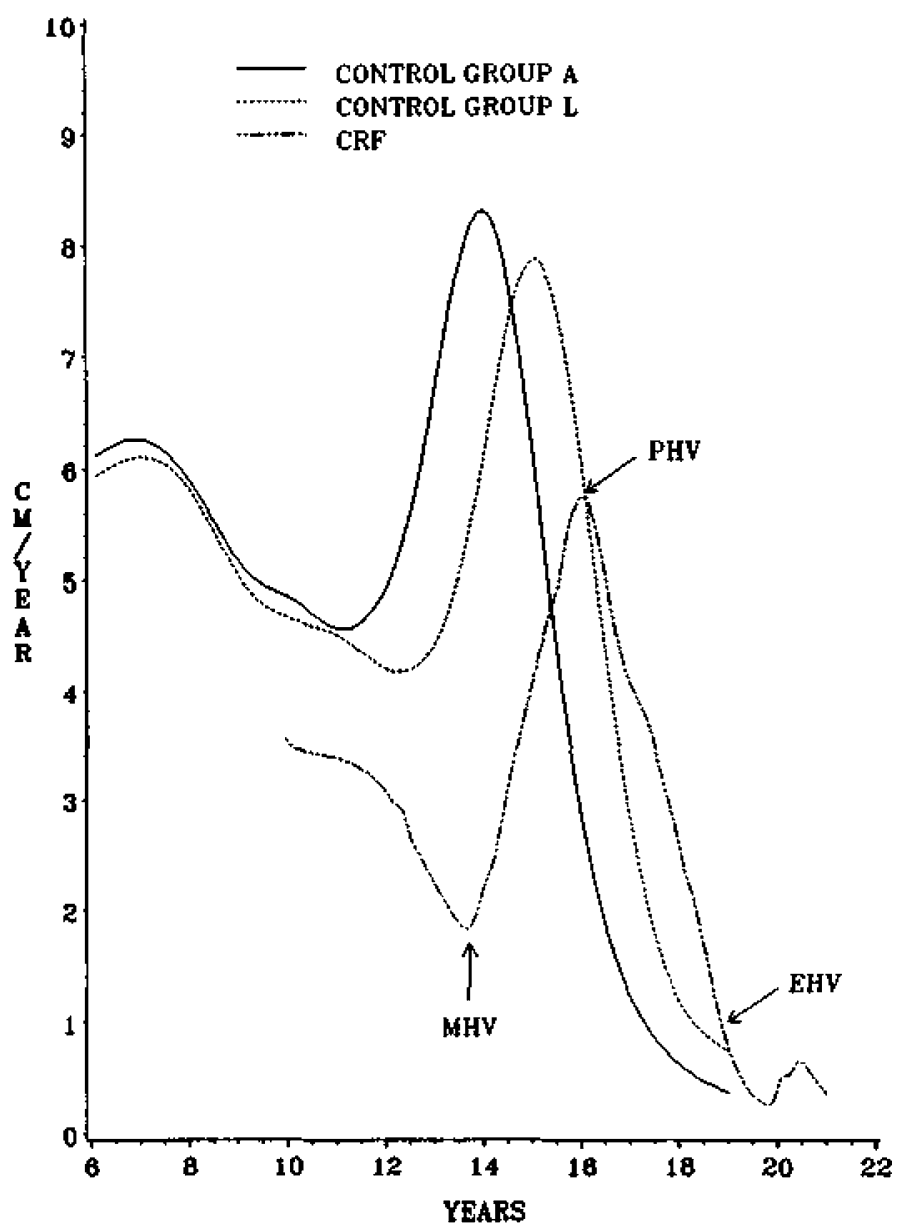

Fig. 2. Synchronized mean height velocity curves of 15 pubertal boys with CRF and healthy children maturing at average (control group A) and late (control group $L$ ) age. 